\title{
EPIDEMIOLOGY AND IMIMUNOPATHOLOGY OF RABIES VIRUS
}

\author{
Waqas Ahmad ${ }^{* 1}$, Muhammad Ahsan Naeem², Muhammad Younus³, Qaiser Akram³ and Qamar-un-Nisa ${ }^{4}$
}

'Department of Clinical Sciences, University College of Veterinary and Animal Sciences Narowal, 5160o, Pakistan ${ }^{2}$ Department of Basic Sciences, University College of Veterinary and Animal Sciences, Narowal, 5160o, Pakistan 3Department of Pathobiology, University College of Veterinary and Animal Sciences, Narowal, 5160o, Pakistan ${ }^{4}$ Department of Pathology, University of Veterinary and Animal Sciences, Lahore, Pakistan

*Corresponding author: waqas.hussain@uvas.edu.pk

\section{INTRODUCTION}

Rabies virus (RABV) belongs to the family Rhabdiviridae, and genus Lyssavirus. This pathogen is oftent cited in literature as a 'cunning virus' because it produces severe neurological signs and symptoms, that ultimately lead to encephalitis and death (Jackson 2010; Mani and Madhusudana 2013). Rabies is an ancient zoonotic disease and a highly underestimated public health problem of the developing world. It is also the most feared infectious disease and a top priority neglected tropical disease of poor and marginalized areas (Hampson et al. 2010; Mani and Madhusudana 2013). Research studies have focused on the host-cell response of RABV, using in vitro and in vivo models to explain how RABV causes neuronal dysfunction instead of cell death. The molecular interface between RABV and the host-cell immune system is relatively complicated as compared with other viruses of different genera. Specific genes of RABV play key roles in attaching the viral coat to the host-cell surface, followed by transcription, translation, and release of new viral particles via clathrin-mediated endocytosis with the help of an actin-microtubule cytoskeleton. Hence, understanding of the virology and specific features associated with RABV genes are integral to envisage the immunological properties and the pathways to produce pathogenesis. In this chapter, epidemiological characteristics of RABV are described to understand spatio-temporal distribution and clinical profiles of this virus. Additionally, important host-cell responses, such as production of interferon, neuronal and mitochondrial dysfunctions, apoptosis, and production of Negri-bodies through evasive strategies of RABV are also discussed.

\section{Historical Perspective of RABV}

The word 'rabies' has been originated from the Greek word 'Lyssa', and it has also been often written in literature as Lussa, Lytta, or Lutta. Another finding reported that the word 'rabies' originated from a Sanskrit or Latin word called Rabbahs or Rabere, meaning 'fierce'. Moreover, the word 'Lyssa' has also been derived from 'Lykos', meaning 'wolf. This idea was dominated during the $30^{\text {th }}$ century $\mathrm{BC}$, which is also known as the Indian Vedic period (Geison 1978; Schneider et al. 1988).

Interestingly, rabies is the most ancient zoonosis of humans and there are many variations and controversies in delineating the actual and precise history of RABV in scientific literature and encyclopedias. The ancient etymologists have reported that the basic origin might have been from 'Lysis', meaning decomposition. However, early scientists used to describe lyssa in various forms, such as furious expressions, madness, or lyrical songs. Hence, an authentic study has not been able to define the literal origin of the word 'rabies'. Hippocrates were the first, who used the already existing word (rabies) to explain the term 'hydrophobia' or fear of water (Steck et al. 1982).

In ancient periods, people believed in many superstitions, such as considering furious dogs as a 'god of death' (Geison 1978; Schneider et al. 1988). A person infected with RABV would eat shearwater liver, gentian, or river crabs as an attempt of postexposure prophylactics to prevent rabies. These bizarre and ignorant remedies were considered as treatment of rabies. In severe cases of dog bites and suspected rabies, cauterization of the victim's skin was also a thoughtful treatment. In brutal and outlandish acts examples, the tongue of a rabid dog was removed and pinched under the foot or toes of an infected victim so that he/she may get rid of this disease. In addition to these, chicken feces were also offered as a remedy to rabies in suspected dogs. If any of these methods had not proved successful, then the victim had to be thrown in shallow water to let him/her drink plenty of water to overcome the signs of rabies (King et al. 2004). Various strange, but unsuccessful, treatments had developed that usually included the manipulations of blood samples from the dog bite wound. The fear of rabies was overwhelming because of unknown multiple reservoirs and the absence of credible treatment (Steck et al. 1982; Dunlop 1996; King et al. 2004).

The first major rabies epizootic was reported among foxes and dogs in North America in 1768. By 1771, the disease had spilled over from the canids to domestic animals and swines (Dunlop 1996). Within a few years, the outbreak sprawled to the regions of France, the West Indies, and eventually penetrated throughout North America. The earliest reliable rabies data were found in the Eshnunna Mesopotamian Codex during 1930 BC. It stated that the suspected owner of a pet dog was supposed to acquire control measures to stop the infection. If a rabid dog caused death to other individuals, then the owner of that rabid dog had to pay for the price of the dead victims. During the late $19^{\text {th }}$ century, almost 1,112 humans died of rabies in England (Dunlop 1996; George 2011).

George Zinke demonstrated for the first time in 1802 that rabies might possibly be transmitted from saliva of an 
infected dog to a healthy dog (Zinke 1804). In 1879, Pierre-Victor Galtier reported the transmission of an actual case of rabies from one animal to another (Galtier 1879). The fear of horrifying deaths began to decline by the end of 1885, when two French scientists, Emile Roux and Louis Pasteur, successfully introduced the first vaccine against RABV on Joseph Meister, a victim of a rabid dog bite. The vaccine had attenuated or weakened RABV particles, which were harvested from the brain of a rabbit that died of rabies. The components of the vaccine were also weakened by keeping the vials under dry conditions for five to ten days (Geison 1999).

\section{Global Epidemiology}

In 2009, raccoons, skunks, and bats were reported to be responsible for $98 \%$ of rabies cases in America (Blanton et al. 2009). At present, dogs are the principal reservoir of RABV and account for 99\% of rabies cases in Asian and African states (Hampson et al. 2015). Globally, rabies causes approximately 59,0oo human deaths, with the highest death toll in Asia (59.6\%), followed by Africa (36.4\%) and America (0.05\%). The economic and social cost values of the prophylactic and control measures are also considerably higher in the developing countries, as shown in Fig. 1 (Hampson et al. 2015). Fig. 1 also shows the highest death rate in Asia, followed by Africa and other countries of the world. Different percentages of costs pertaining to the vaccination, surveillance, animal losses, travel cost, income losses, and premature deaths have also been shown in Fig. 1.

Rabies has influenced almost 150 countries of the globe, and the underdeveloped countries are at the front list. However, few states (such as New Zealand, Japan, Britain, and Australia) have almost eradicated canine rabies by adopting strict serosurveillance and control measures, together with increasing availability of postexposure prophylaxis (Hudson et al. 1996; Mani and Madhusudana 2013). The Caribbean and Latin America have substantially decreased cases of rabies in animals and humans by following the recommended guidelines of the World Health Organization (WHO 2013).

A total of 8,384 rabies cases were recorded in dogs and humans in the United States, which included 33 indigenous cases reported in 1946 (Talbi et al. 2009). In later periods, annual human rabies cases decreased from hundreds to less than a dozen by the end of 2010. Some countries, including Paraguay, Uruguay, Belize, Colombia, Chile, Ecuador, Panama and Costa Rica have not reported any rabies cases in animals nor in humans for the last ten years. Large-scale dog vaccination programs have reduced considerable number of rabies deaths in animals and humans in Indonesia, Sri Lanka, the Philippines and India (Youwen et al. 2016).

The major reasons behind higher death tolls due to rabies in Asia and Africa are climatic conditions, lack of awareness, ignorance towards the use of prophylactic measures, and deficient integrated control strategies at domestic and national levels. Human rabies-related death rates in China are mainly concentrated in the south (Gunagxi, Gunagdong, Hunan, Henan and Hubei provinces), which is a relatively hot region (Yin et al. 2013). In China and neighboring Pakistan, the number of dog bite cases burgeoned higher during summer months (April, May and June). Multiple surveys in these two countries have concluded that the dog bites and incomplete follow-up of prophylactic measures are the major barriers in reducing the concurrent rabies cases (Yin et al. 2013; Zaidi et al. 2013). Kathmandu has previously reported 200 human rabies deaths annually and recorded up to 35,00o stray dogs (Hampson et al. 2015; Tenzin et al. 2015). In Sri Lanka, 100 people die of rabies each year, while in Bangladesh 3,00o human deaths were reported during 2009 (Smith and Rupprecht 2008; Tenzin et al. 2015). In Pakistan, the highest numbers of dog bites were routinely reported in Karachi during 2013, and there were nine people out of one million suffer and die from rabies (Parviz et al. 2004; Zaidi et al. 2013). Figure 2 shows the distribution of rabies cases and costs of various parameters associated with these cases worldwide. Deaths due to rabies may be due to failure in a range of important factors, such as limited dog vaccination, unavailability of travelling cost, animal losses and non-existent surveillance tools etc. The gray area of the Figure also shows countries free from canine rabies, while perhaps some variants of RABV may still have caused sporadic outbreaks of the disease among these canine rabies-free countries (Hampson et al. 2015).

\section{Transmission}

The transmission of RABV has been reported through horizontal and vertical (mother to fetus) routes in domestic and wild animals (Jackson 2000; Jackson 2010). It may normally occur through animal bites, whereas transmission through human bites, non-bite exposures such as open wounds, mucous membranes contaminated with RABV-infected saliva, abrasions, or scratches, has also been reported (Srinivasan et al. 2005; Schnell et al. 2010). Microscopically, the transmission of RABV occurs through transcytosis, which is a "cell to cell" spread at the neuromuscular junction. The saliva contains the RABV particles that contaminate the wound site in animals or humans, and the RABV attaches with the neuromuscular junction through receptors (Mani and Madhusudana 2013). The preparation of the raw meat (for example butchering) poses a greater risk for transmission than eating it. However, consuming poorly cooked and RABV infected meat can also potentially cause rabies in healthy individuals, hence, ensuring properly cooked meat also depletes the chances of rabies transmission in certain cases (Srinivasan et al. 2005). Furthermore, corneal and organ transplants have been documented for the transmission of RABV (Javadi et al. 1996).

Animals or dog bites are the primary causes of rabies transmission in humans and animals, involving the neuromuscular junction (myoneural junction). This junction is a specialized synapse that connects a motor neuron (a neuronal cell in which cell body resides in the spinal cord and lengthy axon projects outside the spinal cord) to muscles for the transmission of electrical impulses, as shown in the diagram (Fig. 3). 


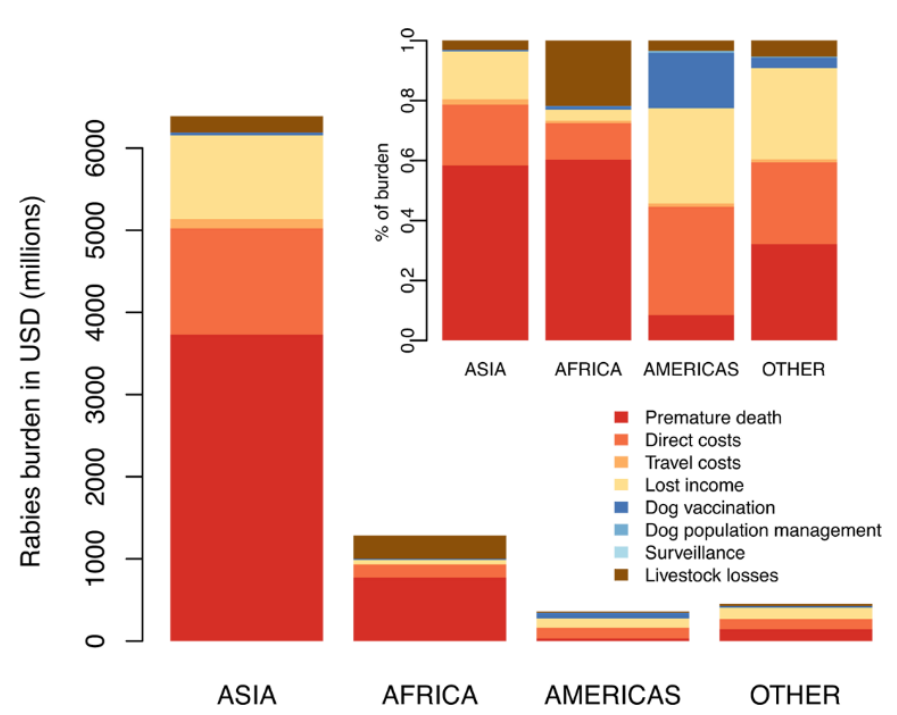

Fig. 1: Rabies burden in USD (millions) across different regions. Inset image shows proportional expenditure in different continents (adopted from Hampson et al. 2015)
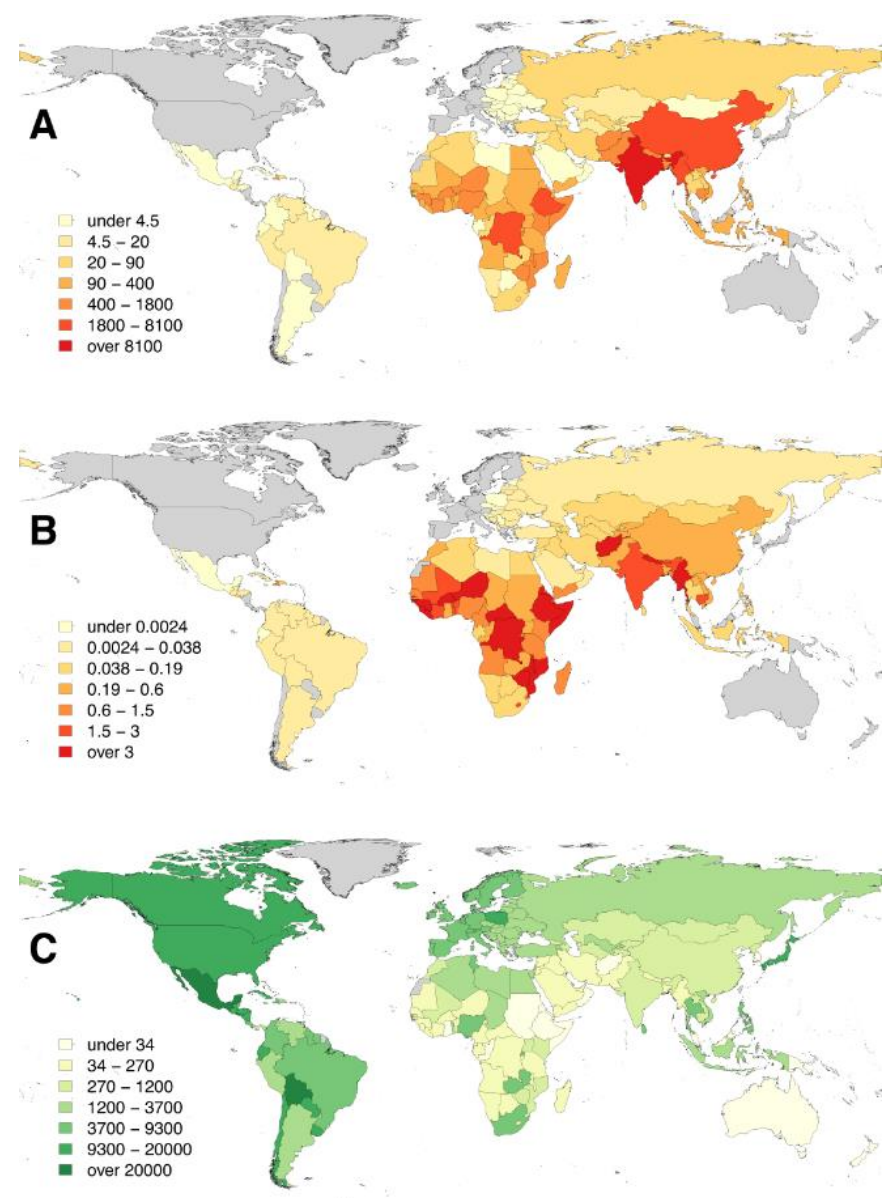

Fig. 2: Rabies burden worldwide: (A) Human deaths due to rabies, (B) Death rates per capita (10o,ooo persons), and (C) cost for dog vaccination (per 100,00o persons). Gray color shows countries where canine rabies has been eliminated (adopted from Hampson et al. 2015)

\section{Reservoirs of RABV}

In the early nineteen-nineties, Dr. Joseph Lennox Pawan first revealed that vampire bats could be the potential source of rabies transmission between animals and

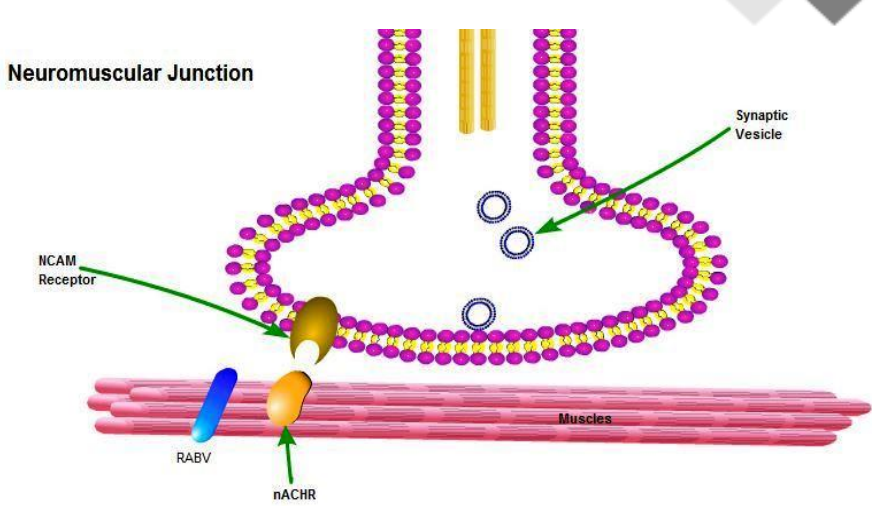

Fig. 3: Schematic representation of neuromuscular junction, showing synaptic terminal, muscle fibres and the corresponding RABV receptors.

humans (Pawan 1936; Pawan 1959). Any warm-blooded mammal can possibly be infected with the RABV by showing the specific symptoms, and is capable of transmitting the disease to humans via bites. Infected bats, skunks, ruminants, caprines, and canines are examples of the wide range of reservoirs disseminating rabies to large territories of the world. Potential exposure to infected farm animals, wild carnivores, and bears are high-risk species, while groundhogs and weasels may also put the host at relatively low risk. Other high-risk groups may include scientists or workers in a rabies laboratory, international travelers, hunters, veterinarians, and pet lovers. Poultry species, small rodents (rats, mice, gerbils, chipmunks, hamsters, squirrels, and guinea pigs) and lagomorphs (rabbits and hares) usually die of bite wounds before they become rabid, but these species can be infected with RABV (Jackson 200o; Jackson 2010; Schnell et al. 2010).

\section{Clinical Profiles}

The incubation period of the RABV depends upon the location of the suspected animal-bite and varies from a few days to weeks, and even months or a year. Generally, it is 20 and 90 days in animals and humans, respectively (Jackson 2000; WHO 2005). In dogs, characteristic symptoms exist with variable intensity, like excessive salivation, tremors, hydrophobia, pharyngeal paralysis, aggression, licking, and restlessness. In addition, sexual excitability, imbalanced movements, hyperexcitibiltiy, excessive urination, and bellowing are commonly seen in domestic and wild animals (Vural et al. 2001; Brookes et al. 2007; Zhang et al. 2011). The clinical signs and symptoms in animals can be divided into three major stages of RABV infection. These are: i) Prodromal phase, ii) Furious/Classical phase, and iii) Numb/Paralytic phase.

\section{Prodromal Phase}

This is the initial stage of the symptoms that appears as soon as the RABV reaches the brain. Intense drooling of saliva and undefined neurological signs appear, leading to death within 10 days. Priapism, numbness, tingling in limb, discomfort or pain at the site of bite, elevated 
temperature, and malaise are the significant clinical signs in humans.

\section{Furious/Classical Phase}

Electrophysiological studies have shown that the furious stage in animals is probably associated with the dysfunction of anterior horn cells of the gray matter of spinal cord and an animal in this phase may die within 1-5 days after onset of symptoms. This phase is characterized by increased viral count in saliva, cardiac arrhythmias, hydrophobia, excitation, convulsions upon exposure to light, air currents, or sound, intense thirst, stages of excitement and hallucination, and extreme aggression. Almost, $80 \%$ of RABV infections in dogs exhibit this phase (Tepsumethanon et al. 2005; Jackson 2010).

\section{Numb/Paralytic Phase}

This form of presentation is characterized by weakness, flaccid paralysis of the limbs and chewing muscles that further spread to all body muscles, resulting in an inevitable death. Almost $20 \%$ of RABV infections in dogs exhibit this phase (Tepsumethanon et al. 2005; Jackson 2010). Clinical signs in the furious and paralytic phases are mainly observed among wild animals (especially dogs). However, rabid animals or humans also show signs of either furious or paralytic phase (sometimes overlap between the two phases have also been observed).

\section{Diagnostic Techniques in Animals and Humans}

Several ante-mortem and post-mortem diagnostic techniques for RABV infection are based on the following three main categories.

\section{Viral Antigens}

This category includes enzyme linked immunoassay, fluorescent antibody test, immunohistochemistry, rapid rabies enzyme immune diagnosis, staining techniques (such as Hematoxylin \& Eosin and Seller's staining for the detection of Negri bodies), direct rapid immunohistohemical test, latex agglutination test, and rapid immunodiagnostic test. Rabies antibodies are used in these tests to detect viral antigens in a suspected sample (Woldehiwet 2005; Stein et al. 2010; Faizee et al. 2011).

\section{Serological Assays}

Fluorescent antibody virus neutralization, virus neutralizing antibody test, and rapid fluorescent focus inhibition test are included in this category (Woldehiwet 2005).

\section{Detection of Viral Genome}

Cell culture inoculation tests, nucleic acid sequencebased amplification, reverse transcriptase polymerase chain reaction, TaqMan RT-PCR, loop-mediated isothermal amplification, and viral isolation through mouse inoculation test are commonly used for detection of RABV infection (Lumlertdacha 2005; Clement et al. 2013).

\section{Prevention and Treatment}

There is no globally accepted treatment to avoid death against rabies once the symptoms of disease appear in humans and animals. Therefore, pre-exposure and postexposure vaccination is highly recommended for humans and animals of high-risk group. Life-support therapy is sometimes practiced in medical wards that contain antagonistic receptors for NMDA interferon- $\alpha$, ketamine, and antiviral drugs (ribavirin) (Jackson 2000). Thus, the use of reliable pre and postexposure prophylactic measures is the last hope to countercheck the pathogenic spread of RABV in animals and humans.

It is worth mentioning here that there are three categories of animal or dog bites inflicted to humans. In the first category, the dog or animal may lick the intact human skin and there is no breakage in the skin. The second category includes abrasions or scratches in the skin without bleeding, while the deep subcutaneous tissue remains intact. The third category of bite involves deep subcutaneous tissue with bleeding, and this type is more serious compared to the previous two categories. These categories of bites do not really 'matter' and anyone with a potential exposure should receive post-exposure treatment for rabies. However, third category bite from a rabid animal is very likely to expose patients to a large viral load, but people have had undetectable small bites from bats or scratches and still succumbed to rabies. In general, the wound site should be immediately washed with soap, and a dose of equine rabies immunoglobulins or human rabies immunoglobulins must be administrated in the third category or severe animal bite for passive immunity. It is mandatory to ensure the complete followup of post exposure vaccination and rabies immunoglobulins, prescribed by the World Health Organization (WHO 2005; Slate et al. 2009).

To produce quality vaccines against rabies, multiple attenuated strains of RABV are used. Amongst these, attenuated rabies vaccine strain SRV9, Pasteur Virus (Dietzschold et al. 2003) and Street-Alabama-Dufferin are well-known (Faber et al. 2002). Pasteur was the first scientist, who isolated RABV from the brain tissue of rabid cow in 1882; this strain of RABV is also used in a parent vaccine of the laboratory-based Challenge Virus Standard (CVS) (Dietzschold et al. 2003). The CVS strain is mostly used in serological assays for the analysis of the antibody titer against different strains of RABV. Currently, different kinds of anti-rabies vaccines are being used in developing and developed countries, such as live attenuated vaccines (oral vaccination) (Borisov et al. 2002), inactivated vaccines (purified chicken embryo cell), human diploid cell rabies vaccine (Dietzschold et al. 2003; WHO Survey of Rabies), DNA vaccines (Bahloul et al. 2003), vector vaccines containing oral vaccines for wildlife (Faber et al. 2002) and plant-derived antigens (Loza-Rubio et al. 2007). 
Table 1: Taxonomic classification of lyssaviruses (adopted from the webpage of 'WHO-Rabies-Bulletin-Europe')

\begin{tabular}{lll}
\hline Virus & Potential vector(s)/reservoirs & Distribution \\
\hline Rabies virus (RABV) & Carnivores (worldwide); bats (Americas) & $\begin{array}{l}\text { Worldwide (except several } \\
\text { islands) }\end{array}$ \\
Lagos bat virus (LBV) & Africa \\
Mokola virus (MOKV) & Rrugivorous bats (Megachiroptera) & Sub-Saharan Africa \\
Duvenhage virus (DUVV) & Insectivorous bats (Microchiroptera) & Southern Africa \\
European bat lyssavirus 1 (EBLV-1) & Insectivorous bats (Eptesicus serotinus) & Europe \\
European bat lyssavirus 2 (EBLV-2) & Insectivorous bats (Myotis daubentonii, M. dasycneme) & Europe \\
Australian bat lyssavirus (ABLV) & Frugivorous/insectivorous bats (Megachiroptera/Microchiroptera) & Australia \\
Aravan virus (ARAV) & Insectivorous bats (Myotis blythi) & Central Asia \\
Khujand virus (KHUV) & Insectivorous bats (Myotis mystacinus) & Central Asia \\
Irkut avirus (IRKV) & Insectivorous bats (Murina leucogaster) & East Siberia \\
West Caucasian bat virus (WCBV) & Insectivorous bats (Miniopterus schreibersi) & Caucasian region \\
Shimoni bat virus (SHBV) & Hipposideros commersoni & East Africa \\
Bokeloh bat lyssavirus (BBLV) & Insectivorous bats (Myotis nattereri) & Europe \\
Ikoma virus (IKOV) & Isolated from Civettictis civetta & Africa \\
Gannoruwa bat lyssavirus (GBLV) & Isolated from Pteropus giganteus & Asia \\
Lleida bat lyssavirus (LLEBV) & Insectivorous bats (Miniopterus schreibersi) & Europe (Spain) \\
\hline
\end{tabular}

Differential diagnosis of RABV can include encephalitis, lockjaw, and epilepsy in the initial phase of infection, while poliomyelitis, belladona poisoning, delerium tremens and acute polyneuritis are with the paralytic phase in humans. Symptoms in hepatitis, distemper epilepsy, brain tumours, poisoning, and head injury can be clinically confused with those in rabid dogs (Dietzschold et al. 2003; Tepsumethanon et al. 2005; Lumlertdacha, 2005).

\section{Taxonomic Classification}

According to the International Committee on Taxonomy of Viruses, RABV falls in Mononegavirales order, Rhabdoviridae family, and Lyssavirus genus. This family further contains Lyssavirus, Ephemerovirus, and Vesiculovirus as genera, while the genus is further subdivided into 11 genotypes based on their antigenic characterization and phylogenic lineage (Table 1). Among these genotypes, classical RABV is commonly found worldwide (Fauquet et al. 2005).

\section{Structure of the RABV}

RABV has a bullet-shaped structure, with a measured length and diameter of $100-300 \mathrm{~nm}$ and $75 \mathrm{~nm}$, respectively (Schnell et al. 2010). The virion measures 12 $\mathrm{kb}$, with two fundamental subunits. The first subunit is the ribonucleoprotein (RNP), which is also associated with phosphoprotein ( $\mathrm{P}$ segment) and viral polymerase, making the internal compact core. The RNP complex comprises nucleoprotein ( $\mathrm{N}$ segment), $\mathrm{P}$ segment, and RNA-dependent RNA polymerase (L segment); these are all collectively associated with viral RNA. The matrix protein ( $\mathrm{M}$ segment) forms the bridge between virion membrane and capsid. The second basic subunit is glycoprotein ( $G$ segment), which wraps around the outer surface of the genome and contains 400 trimeric spiky projections (Tordo et al. 1986; Schnell et al. 2010).

RABV is single-stranded, non-segmented, and negativesense RNA genome of approximately 12,00o nucleotides. The $\mathrm{N}$ segment consists of 450 amino acids and helps in the transcription of RNA. The P segment contains 297 amino acids and is linked to the $\mathrm{L}$ segment to serve as a non-catalytic cofactor for RNA polymerization (Schnell et al. 2010). The $M$ segment comprises 202 amino acids and is responsible for viral assembly and attachment with the host membrane (Mebatsion et al. 1999). An extensively studied protein of RABV is G segment that contains 524 amino acids. This protein is primarily responsible for inducing pathogenic response in the host (Benmansour et al. 1991).

\section{Immunopathology}

The interaction between RABV and the host cell immune system is complicated as compared to other viruses of different genera. Various factors have been discovered to understand the mechanism of rabies immunology. Distinct details about the life cycle and proposed mechanism of rabies endocytosis are discussed below to explain the immunology and pathogenesis of RABV.

\section{Brief Overview of Life Cycle}

It is well known that RABV has three receptors for cellular uptake, namely, nicotinic acetylcholine receptor (nAchR) located at the post-synaptic muscle membrane, neuronal cell adhesion molecule (NCAm) located at the post synaptic membrane of the neuron for the attachment of the RABV, and nerve growth factor P75NTR (BeX 3 and NGFR) which is a molecular structure to provide entry sites for RABV. The life cycle of RABV recirculates the following steps: binding, un-coating, production, and assembly of the viral components inside the host cell (Tuffereau et al. 1998; Thoulouze et al. 1998). Various studies have shown that RABV follows clathrin-mediated endocytosis for the internalization within the endothelial cells (Sun et al. 2005; Piccinotti et al. 2011; Weir et al. 2014).

\section{Internalization}

The precise mechanism to follow the pathways of the clathrin-mediated endocytosis is not yet clear. However, 
many research studies have speculated that viral proteins of RABV are usually internalized through clathrin-coated pits during clathrin-mediated endocytosis, and at later stages in lysosomes using late endosomes. The $\mathrm{G}$ segment of $\mathrm{RABV}$ and $\mathrm{pH}$-dependent membrane fusion are responsible for the membrane fusion and attachment with the host cell (Superti et al. 1984; Pulmanausahakul et al. 2008).

\section{Intracellular Transport}

After the attachment and entry into the host cell, either a single capsid or whole virion is transported to the neuronal cell body of the host cell in the form of a vesicle. During this transport, RABV also interacts with the dynein light chain (LC8), using the support of microtubule along the entire length of axon, but deletion of LC8 alone do not affect the transport of RABV. Another study demonstrated that capsids and enveloped-labeled viruses enabled the transport of the whole virus in endosomal vesicles. The transport mainly depends upon the $G$ segment of RABV, which is embedded inside the vesicle. Thus, the nature of the vesicle determines the transport strategy of RABV (Tan et al. 2007; Klingen et al. 2008).

\section{Replication, Transcription and Translation}

The regulatory mechanism of RABV has evolved for efficient, but limited production of viral particles to avoid recognition by the immune system of the host. Negri bodies, containing ubiquitylated and heat shock protein (Hsp7o), are the entities for the replication and transcription of RABV, and are formed by hijacking the toll-like receptors $\left(\mathrm{TLR}_{3}\right.$ ) (Whelan et al. 2004; Pauline et al. 2009). The interaction of Hsp7o has also been observed with the $\mathrm{N}$ segment of RABV. Viral proteins, mRNAs, and viral particles were reduced as gene expression of Hsp7o protein was down-regulated (Cheng et al. 1983). These findings indicate that Hsp7o has a close association with one or many life stages of RABV assembly, or it may also have a proviral role in the mechanism of RABV pathogenesis. The $M$ segment of RABV also facilitates the process of translation. RABV follows 'stop-start model' to complete its life cycle by starting the transcription at the $3^{\prime}$ end of the RNA and then sequentially synthesis at 5 'end to prepare a genomic ladder of viral protein (Schnell et al. 2010). It has been observed that whenever adequate contents of $\mathrm{N}$ segment are formed through 'stop-start model', the encapsidation of leader RNA forms a complete genome and thus, the phenomena is converted from transcription to replication (Schnell et al. 2010).

\section{Budding}

In the last phase of RABV infection, the $M$ and $G$ segments play their role in the budding, and both viral proteins are interdependent to facilitate the process of budding, as shown in Fig. 4 (Mebatsion et al. 1996). In this stage, the newly formed segments of RABV are assembled and subsequently join to form new virions and release through plasma membrane in the form of budding. Experimental studies in skunks have shown that the budding occurs mostly from synapse or plasma membranes of the dendrites, and less commonly from the plasma membrane of perikaryons (Charlton and Casey 1971). The $M$ and $G$ segments of RABV facilitate the process of budding, and the budding efficiency of RABV decreases 3o-fold in the absence of its G segment (Schnell et al. 2010).

\section{Overview of Clathrin-Mediated Endocytosis}

The internalized vesicle formation follows through the five bulging steps in the clathrin-mediated endocytosis, which proceeds to fuse with early and late endosomes. Figure 4 represents different stages, such as the nucleation, selection of cargo, assembly of coating, scission, and ultimately uncoating of the vesicle. The adaptor protein-2 (AP-2 complex) and Fes/CIP4 homology domain (FCH domains), together with additional factors, accumulate clathrin proteins into a bunch. The clathrin membrane forms curvature to develop a well-defined clathrin coated pit. These changes are also stimulated with the addition of phosphatidylinositol 4, 5,-biphosphate (PIPz) and the whole process is called nucleation (Koh et al. 2007; Dannhauser and Ungewickell 2012).

In this process, the scaffolding protein also plays important role to join the two proteins simultaneously to stimulate the process of molecular signaling. Scaffolding proteins, such as epidermal growth factor receptor substrate 15 (EPS15), and intersectin are also involved in clathrin-meidated endocytosis (Koh et al. 2007; tyszkiewicz et al. 2020). The process of endocytosis is highly complicated, as demonstrated by in vivo studies, while evidence of these scaffolding proteins and other factors has also been described. The adaptor protein-2 also recruits intersectin and EPS 15 that systematically assist the process of clathrin-coated pit formation (Koh et al. 2007; Dannhauser and Ungewickell 2012; Łyszkiewicz et al. 2020). The appendage domain of adaptor protein-2 also binds the corresponding adaptors for the selection of cargo. In addition, the $\mu$ and $\sigma$ sub-units of adaptor protein-2 engage many receptors. This protein forms the clathrin coat around the newly formed clathrin-coated pit. For the scission of the pit, the actin polymerizes at the constricted neck of the clathrin pit (Piccinotti et al. 2011; Weir et al. 2014).

The process of uncoating occurs through the cyclin Gassociated kinase or auxilin. It reveals the clathrin-coated vesicle through heat shock cognate 70 , and releases an independent endocytic vesicle. The clathrin assembly is again ready for the intake of another cargo at the plasma membrane. Different effectors are involved for the induction of curvatures in the flat plasma membrane to form a well-defined invaginated membrane (McMahon and Boucrot 2011). 


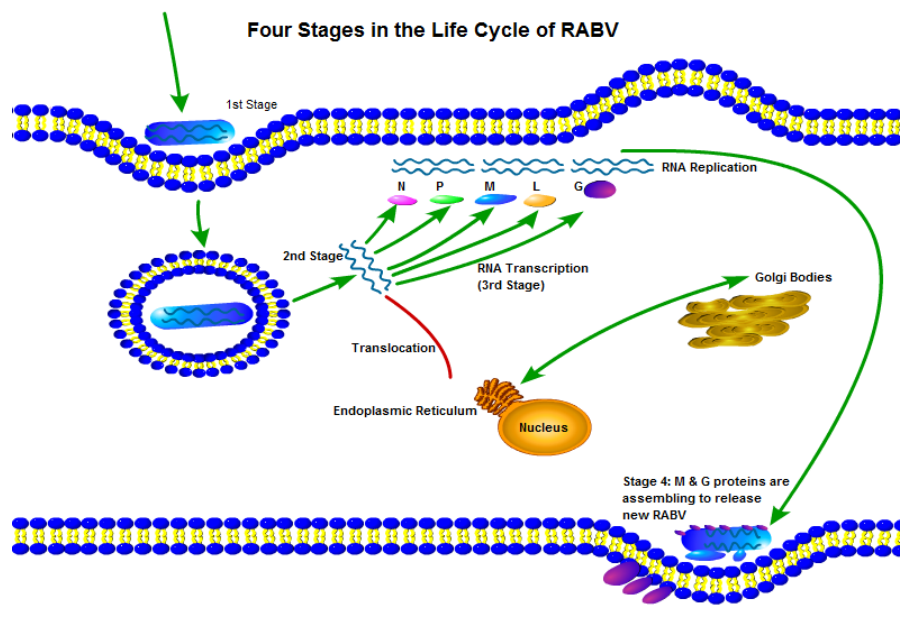

Fig. 4: The first stage includes the attachment and internalization, followed by the genetic expression of different segments of RABV. The third stage is RNA transcription, while the fourth stage includes the assembling of $M$ and $G$ segments of RABV that is again ready to bud out from the cell.

Mechanism of Interferon Production in RABV Infection

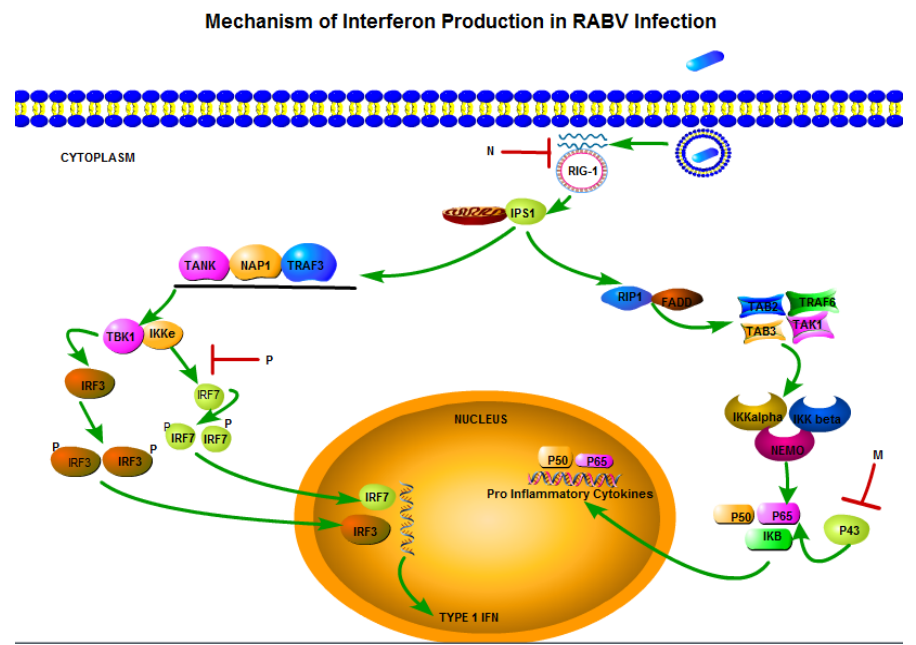

Fig. 5: Schematic mechanism of downstream signaling pathways that start from RIG-1 and further pathways are up-taken by IPS 1 and cystosolic proteins, effector molecules and scaffolding proteins to finally produce type 1 IFN and pro inflammatory cytokines in RABV infection.

\section{Mechanism of Interferon Production in RABV Infection}

The retinoic acid-inducible gene-1 (RIG-1) generally detects the viral RNA, which is a potential activator for the production of type 1 interferons. The RIG-1 is among the most widely observed pattern recognition receptors. It binds to the caspase activation and recruitment domains (CARDs) that facilitate the downstream signaling phenomena. IFN- $\beta$ promoter stimulator I (IPS-1) is usually present on the mictochondrial membrane. The cytosolic proteins detect the presence of RABV segments and bind to the IPS-1 (Fig. 5) to mediate receptor interacting protein-1 (RIP-1), which is also an adaptor protein. The adaptor protein, in turn, phosphorylates with interferon regulatory factor 3 and $7\left(\mathrm{IRF}_{7} / \mathrm{IRF}_{3}\right)$ through TANK-binding kinase 1 $\left(\mathrm{TBK}_{1}\right) / \mathrm{I} \kappa \mathrm{B}$ kinase $(\mathrm{IKK} \varepsilon)$, nucleosome assembly protein 1 $(\mathrm{NAP} 1)$, and tumor necrosis factor-receptor associated factor 3 (TRAF3). This phosphorylated $\mathrm{IRF}_{3} / \mathrm{IRF}_{7}$ is translocated into the nucleus to induce the expression of Type I IFN genes. The adaptor protein complex-1 (AP-1 complex) also interacts with receptorinteracting protein 1 (RIP1) and a death domain called fasassociated protein with death domain (FADD). The latter initiates the activation of the 'nuclear factor kappa-lightchain-enhancer of activated B cells' (NF- $\mathrm{BB}$ ) pathway that is a combination of hetero-and homodimeric complexes of the Rel family members and controls cytokine production and cell survival. Mitogen activated protein kinase kinase kinase (MAPKKK) are important set of signaling proteins for cell differentiations and other intracellular functions. It has cofactor proteins TGF- $\beta$ activated kinasel (TAK1) and its associated binding/activating partners TAK1-binding protein-2 $\left(\mathrm{TAB}_{2}\right)$ and TAK1-binding protein-3 $\left(\mathrm{TAB}_{3}\right)$. The TAK1 is an integral protein to regulate interleukin and activate NF- $\kappa B$ pathway. The TAB2 is a binding or scaffolding protein of TAK1 that connects TAK1 to TRAF6. The $\mathrm{p} 65 / \mathrm{p} 50$ heterodimers are the finest rated subunits of NF$\kappa B$ pathway. A new member of the NF- $\kappa B$ family, p43 (RelAp43), has also been recently recognized. IкB kinase (IKK) is a complex that consists of regulatory subunit NF$\kappa \mathrm{B}$-essential modulator (NEMO) and catalytic kinase subunits (IKK $\alpha$ and/or IKK $\beta$ ). Its activation results in the phosphorylation and subsequence degradation of IкB. This phenomenon helps to release free NF- $\mathrm{BB}$ in translocating to the nucleus, where it induces target gene expression, as well as pro-inflammatory cytokine encoding genes (Fig. 5).

\section{Phosphoprotein of RABV Inhibits Innate Immune Response}

RABV has developed an escape mechanism to skip the trap of the immune system and to avoid the neutralization of virion within the host defense system (Alandijany et al. 2005). The $\mathrm{P}$ segment of RABV counteracts IFN reaction by jamming the response of host cell to type I and II IFN signaling and pathogenassociated molecular patterns (PAMPs). Melanoma differentiation-associated gene $5\left(\mathrm{MDA}_{5}\right)$ and RIG-I are the cytoplasmic helicases that detect the viral RNA. The MDA 5 and RIG-1 are also known as $\mathrm{IFIH}_{1}$ and $\mathrm{DDX}_{5} 8$, respectively (Caillet-Saguy et al. 2015). These innate sensors sensitize or trigger through a TBK1-ІкB kinase $\varepsilon$ (IKKe) complex and NF-kB activator. The NF-kB activator is associated with TRAF family member, and is also termed as TANK. These triggering responses induce IFN regulatory factor $3\left(\mathrm{IRF}_{3}\right)$ phosphorylation (Brzozka et al. 2005). The resultant $\mathrm{IRF}_{3}$ dimerizes itself and is transported to the nucleus to provoke the transcription of interferon beta (IFN $\beta$ ) in concurrence with nuclear factor- $\kappa \mathrm{B}(\mathrm{NF}-\kappa \mathrm{B})$ and activate transcription factor 2 (ATF2). Furthermore, the induction response is also sent by $\mathrm{TLR}_{3}$ receptor. The $\mathrm{P}$ segment of RABV inhibits the IFN $\beta$ by blocking the phosphorylation of $\mathrm{IRF}_{3}$ in the cytoplasm, and it may also block the signal transduction pathways (type I and type II). Normally, Janus kinase 
(JAK) carries out phosphorylation of transcription 1 (STAT1), but $\mathrm{P}$ segment of RABV attaches the phosphorylated STAT1, and inhibits its antiviral transcriptional response and transports to the nucleus (Vidy et al. 2005). Smaller versions of $P$ segment bind the STAT1 and STAT2 heterodimers complexed with IRF9 in the nucleus. This result in the formation of two complexes, STAT1 homodimers and IFN stimulated growth factor 3 (ISFG 3 ). These complexes prevent the activation of IFN-stimulated response element (ISRE), type I and II IFN-dependent immune response, and $\gamma$ activated sequence (GAS)(Vidy et al. 2007).

\section{Immunopathology of RABV}

Rabies pathogenesis is diverse and covers a wide range of diverse lesions, as described comprehensively in the following sections.

\section{Specific Immunopathological Lesions}

Negri bodies are the granular and oval-shaped cytoplasmic inclusion bodies, which are observed in the stained tissue sections processed through paraffin embedded tissue sections. Negri bodies are mostly seen in the RABV-infected purkinje cells of the hippocampus, and often termed as the pathognomonic or characteristic intracytoplasmic bodies to confirm the presence of RABV. These inclusion bodies appear pinkish and magenta red in tissue sectioning and Seller's staining techniques, respectively (Jogai et al. 200o; Schnell et al. 2010; Stein et al. 2010; Faizee et al. 2011). Gross lesions of the brain tissue include congestion, edema, and petechial hemorrhages during post-mortem examination of animals. Likewise, Hematoxylin and Eosin staining technique has shown congestive changes, perivascular cuffing surrounded by multiple layers of inflammatory cells, Babès nodules, vacuolation, rod-shaped neurons, satellitosis, and spongy (cavernous) lesions in naturally and experimentally infected tissues of animals. Similarly, variable proportions of RABV antigens have also been reported in different parts of the brain tissue during diagnosis through immunohistochemistry (Stein et al. 2010; Faizee et al. 2011).

\section{Neuronal Dysfunction}

The RABV causes neuronal dysfunction by manipulating the proapoptotic factors and reducing the threshold level of corresponding gene expression in host cells (Schnell et al. 2010). Disruption in ion channels has already been interpreted in the neuroblostoma cells. The functional impairment in sodium and potassium ion channels is associated with the loss of action potential and membrane polarization (Iwata et al. 1999). The functional proteins of ion channels and the ability to maintain homeostasis are also altered (Dhingra et al. 2007). The Glu81 and Arg77 amino acid sequences of $M$ segment of RABV regulate the survival of infected neurons, and these amino acid sequences induce apoptosis (Bauer et al. 2015). The RABV also causes cell death, either by producing necrotic changes or apoptosis. The CVS strain of RABV caused neuronal apoptosis in mouse neuroblastoma cells, while attenuated viruses caused apoptotic changes in primary neuronal cultures as compared with virulent strains (Theerasurakarn and Ubol 1998; Morimoto et al. 1999; Fazakerley and Allsopp 2001).

\section{RABV Induces Apoptosis}

Many strains of RABV induce apoptosis, but pathogenic viral strains are not included in this category (Jackson 1998). Human lymphocytes and mice showed apoptotic changes with the highly attenuated RABV strain called ERA, but these findings have not been reported in naturally infected humans (Thoulouze et al. 1997). Highly attenuated RABV strains produce more apoptotic changes as compared to the pathogenic strains because of increased production of $G$ segmented protein of RABV (Hemachudha et al. 2005).

\section{Degeneration of Neuronal Processes}

RABV is also responsible for the degeneration of neuronal processes, as well as disintegration of synaptic structures, in the hippocampus of mice ( $\mathrm{Li}$ et al. 2005). The MRV strain of RABV induces dendritic injuries and depolymerization of actin filaments in the hippocampal cells of mice (Yan et al. 2013).

\section{Configuration of Negri Bodies}

There are almost 10 different kinds of TLRs in humans. These are present on the cell surface, act as innate immune receptors, and respond to PAMPS program (Rock et al. 1998). In the formulation process of these intracytoplasmic Negri bodies, RABV hijacks the TLR3 receptor. These are present in the central nervous system and high levels are also found in glial cells (Jack et al. 2005). The Negri bodies are in fact composite of 'aggregated TLR3' found in an inner core that is enclosed by a cover of genomic material and proteins. Negri bodies are not observed in $\mathrm{TLR}_{3}$ deficient experiments, hence the rate of RABV growth decreases significantly. Furthermore, they are also located in late endosomes in human's neuronal cells, as shown in Fig. 6 (Pauline et al. 2009).

Mechanism of Mitochondrial Dysfunction and Oxidative Stress

The CVS infection results in mitochondrial dysfunction that further induces degeneration of neuronal processes, oxidative stress and over-production of reactive oxygen species. In mitochondria of RABV infected neurons, the activities of electron transport chain (ETS) complex I and IV are enhanced. This could be due to three distinct reasons; a) direct or indirect interaction with RABV proteins, b) consequences produced by the up-regulation of sirtuin activity, c) higher $\mathrm{NADH} / \mathrm{NAD}^{+}$ratio. The sirtuins are group of protein family that control metabolism, stress responses, longevity and development. 


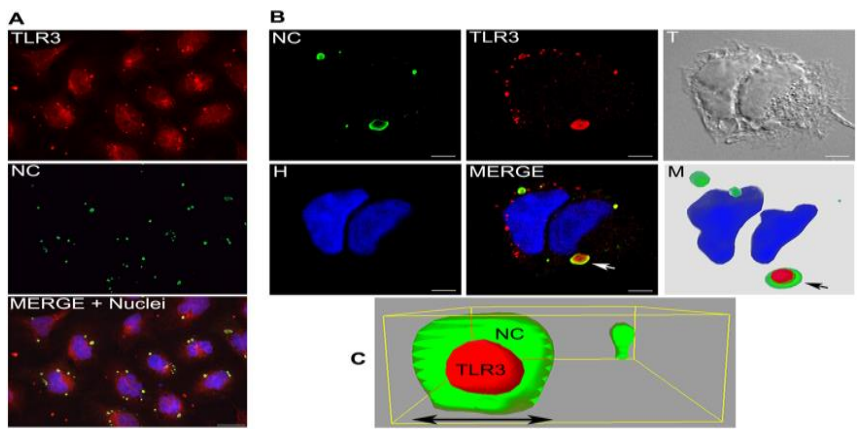

Fig. 6: Diagram showing the TLR3 and $N$ segment of RABV in the perinuclear structures. Nucleocapsid (NC) is green, TLR3 is red and nuclei are blue; The insets $(M \& C)$ show the $3 D$ image of Negri bodies with an internal aggregation of $\mathrm{TLR}_{3}$ (red) enclosed by NC with a diameter of $2.7 \mu \mathrm{m}$ (adopted from Pauline et al. 2009).

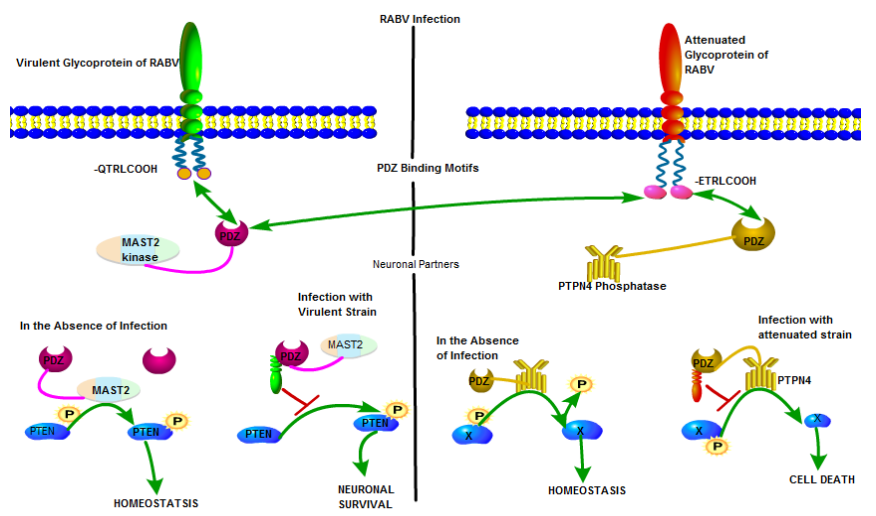

Fig. 7: Schematic diagram showing different neuronal factors and cellular signaling pathways to hijack PDZ domain by virulent (left) and attenuated (right) strains of RABV.

The prompt propelling of protons across the mitochondrial membranes produces a high mitochondrial membrane potential. Electron leakage during forward and reverse ETS complexes also results in superoxide formation which is dismutated by mitochondrial superoxide dismutase into hydrogen peroxide. Superoxide and hydrogen peroxide reduce the levels of intracellular ATP by raising hydrolytic levels of ATP molecules. Mainly, the accumulation and excessive production of superoxide and hydrogen peroxide cause oxidative stress that ultimately leads to degenerated neuronal processes (Alandijany et al. 2005).

Oxidative stress also plays a vital role in dendritic beading, impaired axonal growth, and injury of dorsal root ganglionic neurons due to CVS-11 strain of RABV. The immuno-staining showed that axonal outgrowth was reduced at certain spots of neurons, and modified shapes (kidney and circular) of axonal swellings were seen (Jackson, 2010).

Interferon Stimulated Genes (ISGs) Inhibits RABV Life Cycle

IFN-inducible transmembrane proteins inhibit RABV entry into host cells. These are diverse proteins that have been remarkably efficient in blocking entries of Influenza and Dengue viruses (Siegrist et al. 2011). The cholesterol25-hydroxylase is an endoplasmic reticulum-linked enzyme that manipulates the action of viral fusion with the cell by inducing changes in the cellular membrane (Holmes et al. 2011). MxA is a gene product of two human genes Mx1 and Mx2 that blocks primary transcription (Verhelst et al. 2013). The ISG20 is a $20 \mathrm{kDa}$ protein that plays a key role in host defense, whereas ProMyelocytic Leukemia (PML/TRIM19/Motif protein-19) induces protein degradation and antiviral defense (Nisole et al. 2005). Both of these ISGs inhibit secondary mode of transcription. Protein kinase and IFN-inducible proteins with tetratricopeptide repeats of proteins inhibit viral translation, while virion cellular budding is inhibited by Tetherin (Danielle et al. 2015).

\section{Evasive Strategies in RABV Infection}

RABV promotes neuronal survival and prevents premature cell death of the infected neurons through three important mechanisms. First, the TLR 3 prevents axonal growth by residing within the nucleo-core of RABV and in this way, enables survival of infected neurons. Secondly, a study investigated the attenuated or virulent strain of RABV and unleashed the underlying involvement of PDZ domains that represent a family of protein-protein domains interaction in cells. The PDZ is an acronym made up from three diverse domains, namely the Post synaptic density protein, Drosophila disc large tumor suppressor, and Zonula occludens-1 protein. The PDZ domains regulate multiple vital functions, like ionic transport, signal transduction pathways and cellular signaling, in various species and their number vary from specie to specie. These domains mainly control the cellular functions, such as neuronal signal transmission, and recognize PDZ-binding motifs (PBM) that are usually found at the carboxyl terminus of proteins. The RABV mimics the host PBM and hijacks the PDZ proteins to manipulate host cellular functions to complete one or more stages of its life cycle. The survival or death ratio in infected neuroblastoma cells is regulated by a single mutation $Q(-3)$ E in the C-terminal cytoplasmic tail of $G$ domain, which is termed as CytoG (Prehaud et al. 2010). This mutation is found in a PBM at the C-terminus sequence of CytoG and abundantly influences the nature of the neuronal allies interacting with $G$ domain. In case of virulent (left side in Fig. 7) and attenuated strain (right side in Fig. 7) infection, the viral $G$ segment with theQTRLCOOH PBM and ETRLCoOH PBM interacts with the PDZ domain of microtubule-associated serine and threonine kinase-2 (MAST2). However, -ETRLcoon PBM interaction also involves other cellular partners, such as tyrosine-phosphatase $\left(\mathrm{PTPN}_{4}\right)$. In uninfected neurons, PTEN phosphorylates by MAST2, but the G domain interacts via its PBM with the PDZ domain of the MAST2 in virulent strain infection. This contact may possibly stop MAST2-controlled phosphorylation of PTEN, which changes the homeostasis of the RABV-infected cells and survives the infected cell. The cellular partner $\mathrm{X}$ (as shown in right side of Fig. 7) is dephosphorylated by 
PTPN $_{4}$ in the absence of infection, but the viral PBM interacts with the PDZ domain of $\mathrm{PTPN}_{4}$ in case of an attenuated viral strain infection. This interaction controls proficient dephosphorylation of cellular partner $\mathrm{X}$ that modifies the homeostasis of the infected cell and triggers apoptosis, as shown in Fig. 7 (Prehaud et al. 2010).

Thirdly, the interferon beta production, TLR signaling, and RIG-I induce the expression of FasL, HLA-G and B7$\mathrm{H}_{1}$ proteins in RABV infection. These biological components move to the surface of non-infected astrocytes or infected neurons. At these sites, these molecules interact with their respective receptors on $\mathrm{T}$ cells (CD8 for HLA-G, Fas for FasL and PD-1 for B7-H1). These complex molecules or ligands further trigger the death of $\mathrm{CD}^{+} \mathrm{T}$ or $\mathrm{CD}_{3}{ }^{+}$cells that facilitate the entry of $\mathrm{RABV}$ in the nerve tissue. It is the critical step for the progression of the disease in the nervous system of the host. Due to these three strategies, it is a relatively complicated fight against the well-automated evasion mechanism of RABV (Larrous et al. 2010; Lafon 2011).

\section{Conclusions}

Rabies has remained as the top priority neglected tropical disease of humans by the World Health Organization since ancient period. Historical findings have proven that lack of awareness, ignorance and weird curative methods have created an everlasting fear in poor communities of the developing world. Knowledge regarding epidemiology, zoonotic potential, and pathogenesis of RABV are imperative ways to understand the biology and mode of viral actions in different species. Rabies endemic countries have to spend designated budget on control programs, such as national mass dog vaccination, to attain the goal of rabies elimination by the end of 2030. It would definitely require highly coordinated and collaborative efforts of multiple stakeholders and health professionals of the respective countries.

Insights into immunology, pathobiology, and evasive techniques of RABV are useful to understand the real time infectious pathways of RABV and to design effective vaccines or chemical ligands to block the receptormediated endocytosis inside host cells. RABV has shown well-known pathological changes inside host cells that include, specific pathognomonic lesions, neuronal dysfunctions, apoptosis, neuronal degeneration, alteration in the neuronal cytoskeleton, and formation of Negri bodies. All these pathological alterations enable the RABV to survive inside the complex host-cell environment and produce featured clinical signs and symptoms in multiple species. RABV is a cunning virus that adopts sophisticated pathways to dodge the immune system of the host cells. Latest tools of real time live imaging and intrinsic factors of immune system may help to solve the mystical pathogenesis of RABV.

\section{REFERENCES}

Alandijany et al., 2005. Mitochondrial dysfunction in rabies virus infection of neurons. Journal of Neurovirology 19: 537-549.
Bahloul et al., 2003. Post-exposure therapy in mice against experimental rabies: A single injection of DNA vaccine is as effective as five injections of cell culture-derived vaccine. Vaccine 22: 177-184.

Bauer et al., 2015. A Dynein light chain 1 binding Motif in rabies virus polymerase $\mathrm{L}$ protein plays a role in microtubule reorganization and viral primary transcription. Journal of Virology 89: 9591-960o.

Benmansour et al., 1991. Antigenicity of rabies virus glycoprotein. Journal of Virology 65: 4198-4203.

Blanton et al., 2009. Rabies surveillance in the United States during 2009. Journal of Veterinary Medicine 237: 646-657.

Borisov et al., 2002. Efficacy and safety testing of the viral vaccine "Sinrab" on the targeted species. Scientific Notes of Vitebsk State Academy of Veterinary Medicine. Vitebsk 38: 15-18.

Brookes et al., 2007. Susceptibility of sheep to European bat lyssavirus type-1 and -2 infection: A clinical pathogenesis study. Veterinary Microbiology 125: 210-223.

Brzozka et al., 2005. Identification of the rabies virus $\alpha / \beta$ interferon antagonist: Phosphoprotein $\mathrm{P}$ interferes with phosphorylation of interferon regulatory factor 3. Journal of Virology 79: 7673-7681.

Caillet-Saguy et al., 2015. Strategies to interfere with PDZmediated interactions in neurons: What we can learn from the rabies virus. Progress in Biophysics and Molecular Biology 119: 53-59.

Charlton KM and Casey GA, 1971. Experimental rabies in skunks-immunofluorescence light and electronmicroscopic studies. Laboratory Investigations 41: 36-44.

Cheng et al., 1983. Actin polymerization and synthesis in cultured neurons. Experimental Cell Research 147: 303-314.

Dannhauser PN and Ungewickell EJ, 2012. Reconstitution of clathrin-coated bud and vesicle formation with minimal components. Nature Cell Biology 14: 634-639.

Danielle et al., 2015. Resistance to Rhabdoviridae infection and subversion of antiviral responses. Virus 7: 3675-3702.

Dhingra et al., 2007. Proteomic profiling reveals that rabies virus infection results in differential expression of host proteins involved in ion homeostasis and synaptic physiology in the central nervous system. Journal of Neurovirology 13: 107-117.

Dietzschold et al., 2003. New approaches to the prevention and eradication of rabies. Expert Reviews of Vaccine 2: 89-96.

Dunlop, 1996. Veterinary Medicine: An Illustrated History. Mosby.

Faber et al., 2002. Overexpression of the rabies virus glycoprotein results in enhancement of apoptosis and antiviral immune response. Journal of Virology 76 : 3374-3381.

Faizee et al., 2011. Pathological, immunological and molecular diagnosis of rabies in clinically suspected animals of different species using four detection techniques in Jordan. Transboundary and Emerging Diseases 1865: 1-11.

Fauquet et al., 2005. Virus Taxonomy, Classification and Nomenclature of Viruses: The eighth Report of the 
International Committee on Taxonomy of Viruses. Part II-The Negative Sense Single Stranded RNA Viruses. Ball LA (Eds.). Elsevier Academic Press, pp. 6o9-614.

Fazakerley JK and Allsopp TE, 2001. Programmed cell death in virus infections of the nervous system. Current Topics in Microbiology and Immunology 253: 95-119.

Galtier V, 1879. Etudes sur lza rage. Annales de Médecine Vétérinaire 28: 627-639.

Geison GL, 1978. "Pasteur's work on rabies: Reexamining the ethical issues. Hastings Center Report 8: 26-33.

Geison GL, 1999. Pasteur's work on rabies: Reexamining the ethical issues diagnosis for developing countries. Hastings Center Report 26: 1-30.

George G, 2011. The Natural History of Rabies. CRC Press.

Hampson et al., 2015. Estimating the global burden of endemic canine rabies. PLoS Neglected Tropical Diseases 1-20.

Hemachudha et al., 2005. Pathophysiology of humanparalytic rabies. Journal of Neurovirology 11: 93-100.

Holmes et al., 2011. Genomics and proteomics of vertebrate cholesterol ester lipase (LIPA) and cholesterol 25-hydroxylase $\left(\mathrm{CH}_{25} \mathrm{H}\right)$. Biotechnology 1 : 99-109.

Hudson et al., 1996. Clinical features of experimentally induced rabies in cattle and sheep. Zentralbl Veterinarmed 43: 85-95.

Iwata et al., 1999. Modification of membrane currents in mouse neuroblastoma cells following infection with rabies virus. Brazillian Journal of Pharmacology 126: 1691-1698.

Jack et al., 2005. TLR signaling tailors innate immune responses in human microglia and astrocytes. Journal of Immunology 175: 4320-4330.

Jackson AC, 200o. Update on rabies. Research and Reports in Tropical Medicine 2: 31-43.

Jackson et al., 2010. Role of oxidative stress in rabies virus infection of adult mouse dorsal root ganglion neurons. Journal of Virology 84: 4697-4705.

Jackson AC and Park H, 1998. Apoptotic cell death in experimental rabies in suckling mice. Acta Neuropathology 95: 159-164.

Jackson AC, 2010. Rabies pathogenesis update. Revista Pan-Amazônica de Saúde 1: 167-172.

Javadi et al., 1996. Transmission of rabies by corneal graft. Cornea 15: 431-433.

Jogai et al., 200o. Immunohistochemical studies of human rabies. Neuropathology 20: 197-203.

King et al., 2004. Historical Perspective of Rabies in Europe and the Mediterranean Basin; A testament to rabies. OIE World organization for animal health Editions. ISBN: 92-9044-639-0.

Koh et al., 2007. Eps15 and Dap16o control synaptic vesicle membrane retrieval and synapse development. Journal of Cell Biology 178: 309-322.

Klingen et al., 2008. Double labeled rabies virus: Live tracking of enveloped virus transport. Journal of Virology 82: 237-245.

Lafon M, 2011. Evasive strategies in rabies virus infection. Advances in Virus Research 79: 33-53.
Larrous et al., 2010. Two overlapping domains of a lyssavirus matrix protein that acts on different cell death pathways. Journal of Virology 84: 9897-9906.

Łyszkiewicz et al., 2020. Human FCHO1 deficiency reveals role for clathrin mediated endocytosis in development and function of $\mathrm{T}$ cells. Nature Communications 11: 1031.

Li et al., 2005. Degeneration of neuronal processes after infection with pathogenic, but not attenuated, rabies viruses. Journal of Virology 79: 10063-10068.

Loza-Rubio et al., 2007. Development of an edible rabies vaccine in maize, using Vnukovo strain. Towards the elimination of Rabies in Eurasia. Abstract Book International Conference Paris, 27-30 May.

Lumlertdacha, 2005. Laboratory techniques for rabies diagnosis in animals at QSMI. Journal of the Medical Association of Thailand 88: 550-553.

Mani RS and Madhusudana SN, 2013. Laboratory iagnosis of human rabies: Recent advances. The Scientific World Journal pp: 569712 .

McMahon HT and Boucrot M, 2011. Molecular mechanism and physiological functions of clathrin-mediated endocytosis. Nature 12: 518-532.

Mebatsion et al., 1996. Budding of rabies virus particles in the absence of the spike glycoprotein. Cell 84: 941-951.

Mebatsion et al., 1999. Matrix protein of rabies virus is responsible for the assembly and budding of bulletshaped particles and interacts with the transmembrane spike glycoprotein. Journal of Virology 73: 5673-5679.

Morimoto et al., 1999. Pathogenicity of different rabies virus variants inversely correlates with apoptosis and rabies virus glycoprotein expression in infected primary neuron cultures. Journal of Virology 73: 510-518.

Nisole et al., 2005. TRIM family proteins: Retroviral restriction and antiviral defense. Nature Reviews in Microbiology 3: 799-8o8.

Parviz et al., 2004. Rabies deaths in Pakistan: Results of ineffective post-exposure treatment. International Journal of Infectious Diseases 8: 346-352.

Pauline et al., 2009. Toll-like receptor 3 (TLR3) plays a major role in the formation of rabies virus Negri bodies. PLoS Pathogens 5: 1000315.

Pawan JL, 1959. Rabies in the Vampire bat of Trinidad with special reference to the clinical course and the latency of infection. Annals of Tropical Medicine and Parasitology 30: 1-10.

Pawan JW, 1936. Transmission of the paralytic rabies in Trinidad of the Vampire bat: Desmodus rotundus murinus Wagner, 1840. Annals of Tropical Medicine and Parasitology 30: 137-156.

Piccinotti et al., 2011. Uptake of rabies virus into epithelial cells by Clathrin-mediated endocytosis depends upon actin. Journal of Virology 87: 11637-11647.

Prehaud et al., 2010. Attenuation of rabies virulence: Takeover by the cytoplasmic domain of its envelope protein. Science Signaling 3: 5-19.

Pulmanausahakul et al., 2008. The glycoprotein and the matrix protein of rabies virus affect pathogenicity by regulating viral replication and facilitating cell-to-cell spread. Journal of Virology 82: 2330-2338. 
Clement et al., 2013. Rabies virus. In: Mononegaviruses of Veterinary Importance: Pathobiology and Molecular Diagnosis.

Rock et al., 1998. A family of human receptors structurally related to Drosophila Toll. Proceedings of the National Academy of Sciences of the United States of America 95: 588-593.

Schneider et al., 1988. Current oral rabies vaccination in Europe: An interim balance. Reviews of Infectious Diseases 10: 654-659.

Schnell et al., 2010. The cell biology of rabies virus: Using stealth to reach the brain. Nature Reviews Microbiology 8: 51-61.

Siegrist et al., 2011. The small interferon-induced transmembrane genes and proteins. Journal of Interferon and Cytokine Research 31: 183-197.

Slate et al., 2009. Oral rabies vaccination in North America: Opportunities, complexities, and challenges. PLoS Neglected Tropical Diseases 3: e549.

Smith JS and Rupprecht CE, 2008. Rabies in Sri Lanka: Splendid isolation Susilakanthi Nanayakkara. Emerging Infectious Diseases 9: 1-3.

Srinivasan et al., 2005. Transmission of rabies virus from an organ donor to four transplant recipients. New England Journal of Medicine 352: 1103-1111.

Steck et al., 1982. Oral immunization of foxes against rabies: A field study. Zentralblat Veterinätmedica 29: 372-396.

Stein et al., 2010. Immunohistochemical study of rabies virus within central nervous system of domestic and wild life species. Journal of Veterinary Pathology 47: $630-636$.

Sun et al., 2005. Role of clathrin-mediated endocytosis during vesicular stomatitis virus entry into host cells. Virology 338: 53-60.

Superti et al., 1984. Mechanism of rabies virus entry into CER cells. Journal of General Virology 65: 781-789.

Talbi et al., 2009. Evolutionary history and dynamics of dog rabies virus in western and central Africa. Journal of General Virology 90: 783-791.

Tan et al., 2007. The dynein light chain 8 binding motif of rabies virus phosphoprotein promotes efficient viral transcription. Proceedings of the National Academy of Sciences of the United States of America 104: 72297234 .

Tenzin et al., 2015. Dog population estimation and status of the dog population management and rabies control program in Dhaka City, Bangladesh. PLoS Neglected Tropical Diseases 9: ooo3784.

Tepsumethanon et al., 2005. Six criteria for rabies diagnosis in living dogs. Journal of the Medical Association of Thailand 88: 419-422.

Theerasurakarn S and Ubol S, 1998. Apoptosis induction in brain during the fixed strain of rabies virus infection correlates with onset and severity of illness. Journal of Neurovirology 4: 407-414.

Thoulouze et al., 1997. Rabies virus infects mouse and human lymphocytes and induces apoptosis. Journal of Virology 71: 7372-7380.

Thoulouze et al., 1998. The neural cell adhesion molecule is a receptor for rabies virus. Journal of Virology 72: 7181-719o.

Tordo et al., 1986. Walking along the rabies genome: Is the large G-L intergenic region a remnant gene? Proceedings of the National Academy of Sciences of the United States of America 83: 3914-3918.

Tuffereau et al., 1998. Low-affinity nerve-growth factor receptor $\left(\mathrm{P}_{75} \mathrm{NTR}\right)$ can serve as a receptor for rabies virus. EMBO Journal 17: 7250-7259.

Verhelst et al., 2013. Antiviral gatekeepers that restrain the uninvited. Microbiology and Molecular Biology Reviews 77: 551-566.

Vidy et al., 2005. Rabies virus $\mathrm{P}$ protein interacts with STAT1 and inhibits interferon signal transduction pathways. Journal of Virology 79: 14411-14420.

Vidy et al., 2007. The nucleocytoplasmic rabies virus $\mathrm{P}$ protein counteracts interferon signaling by inhibiting both nuclear accumulation and DNA binding of STAT1. Journal of Virology 81: 4255-4263.

Vural et al., 2001. Immunohistochemical and histopathological studies of fixed rabies virus in goats. Journal of Veterinary Research 68: 83-89.

Weir et al., 2014. Host cell virus entry mediated by asutralian bat lyssavirus $G$ envelop glycoprotein occurs through a clathrin-mediated endocytosic pathway that required actin and Rab5. Virology Journal 11: 1-10.

Whelan et al., 2004. Transcription and replication of nonsegmented negative-strand RNA viruses. Current Topics in Microbiology and Immunology 283: 61-119.

$\mathrm{WHO}$, 2013. global vaccine research forum: Epidemiology of rabies in Asia [R]. [http://www.who.int/entity/ vaccine_research/about/gvrf/en/index.html].

World Health Organization 2005. WHO Expert Consultation on Rabies: First Report. Geneva: World Health Organization; 2005.

Woldehiwet Z, 2005. Clinical laboratory advances in the detection of rabies virus. Clinica Chimica Acta 351: 49-63.

Yan et al., 2013. Street rabies virus causes dendritic injury and F-actin depolymerization in the hippocampus. Journal of General Virology 94: 276-283.

Yin et al., 2013. Challenges and needs for China to eliminate rabies. Infectious Diseases of Poverty 2: 1-10.

Youwen et al., 2016. Rabies virus phosphoprotein interacts with ribosomal protein L9 and affects rabies virus replication. Virology 488: 216-224.

Zaidi et al., 2013. Geographic variation in access to dogbite care in Pakistan and risk of dog-bite exposure in Karachi: Prospective surveillance using a low-cost mobile phone system. PLoS Neglected Tropical Diseases 7: 1-13.

Zhang et al., 2011. Diagnosis and molecular characterization of rabies virus from a buffalo in China: A case report. Journal of Virology 8: 101-105.

Zinke G, 1804. Neue Ansichten der Hundswuth, ihre Ursachen und Folgen, nebst einer sicheren Behandlungsart von tollen Tieren gebissenen Menschen. Published by Jena: C.E. Gabler. Externalidentifier. urn:oclc:record:956546387. 